\title{
Construção de produtos educacionais sobre o uso racional de medicamentos
}

\author{
Construction of educational products on the rational use of medicines \\ Construcción de productos educativos sobre uso racional de medicamentos
}

Recebido: 24/10/2021 | Revisado: 04/11/2021 | Aceito: 07/11/2021 | Publicado: 11/11/2021

\author{
Ana Renata Lima Leandro \\ ORCID: https://orcid.org/ 0000-0003-2707-0730 \\ Universidade Estadual de Ciências da Saúde de Alagoas, Brasil \\ E-mail: releandro@gmail.com \\ Cinthya Rafaella Magalhães da Nóbrega Novaes \\ ORCID: https://orcid.org/0000-0003-4795-6328 \\ Universidade Estadual de Ciências da Saúde de Alagoas, Brasil \\ E-mail: rafamn20@yahoo.com.br \\ Érica Paula Barbosa \\ ORCID: https://orcid.org/0000-0002-8280-4699 \\ Universidade Estadual de Ciências da Saúde de Alagoas, Brasil \\ E-mail: erica.barbosa@ academico.uncisal.edu.br \\ Paulla Valéria de Souza Meneses \\ ORCID: https://orcid.org/0000-0001-6749-9377 \\ Universidade Estadual de Ciências da Saúde de Alagoas, Brasil \\ E-mail: paullavaleria@ hotmail.com \\ Melissa Ramos Barros Sales \\ ORCID: https://orcid.org/0000-0002-1842-8473 \\ Secretaria Municipal de Saúde de Arapiraca, Brasil \\ E-mail: melissabsales@hotmail.com \\ Heloisa Helena Motta Bandini \\ ORCID: https://orcid.org/0000-0002-7320-2637 \\ Universidade Estadual de Ciências da Saúde de Alagoas, Brasil \\ E-mail: heloisabandini@gmail.com
}

\begin{abstract}
Resumo
O presente artigo tem como objetivo relatar a experiência da construção de produtos educacionais capazes de informar aos usuários e profissionais da saúde sobre o Uso Racional de Medicamentos, por meio de um estudo descritivo, com abordagem qualitativa, do tipo relato de experiência, decorrente da produção do Mestrado Profissional em Ensino na Saúde e Tecnologia, de uma Instituição de Ensino Superior em Alagoas. A construção de produtos educacionais em saúde é uma das estratégias de ensino empregadas ao longo do curso. Como produto do trabalho, foi elaborada uma série de três vídeos sobre o Uso Racional de Medicamentos. O processo de elaboração desses produtos educacionais tomou como base o método CTM3, através do qual foram exploradas as ferramentas: Análise Transacional, Exploração Sensorial e a Neurolinguística. O vídeo como ferramenta de ensino constitui um instrumento didático que estimula uma educação mais acessível e de fácil comunicação. Desse modo, conclui-se que, na produção de produtos educacionais em saúde, as habilidades, competências e reconhecimento das necessidades sociais são primordiais, assim como o uso das tecnologias da informação e comunicação nas mais diversas formas. O Uso Racional de Medicamentos é um tema relevante e com carência em ampla divulgação.
\end{abstract}

Palavras-chave: Uso racional de medicamentos; Produtos educacionais; Educação em saúde; Ensino.

\begin{abstract}
This article aims to report the experience of building educational products capable of informing users and health professionals about the Rational Use of Medicines, through a descriptive study, with a qualitative approach, of the experience report type, resulting from the production of the Professional Master's Degree in Education in Health and Technology at a Higher Education Institution in Alagoas. The construction of educational health products is one of the teaching strategies used throughout the course. A series of three videos on the Rational Use of Medicines was created. The elaboration process of these educational products was based on the CTM3 method, through which the following tools were explored: Transactional Analysis, Sensory Exploration and Neurolinguistics. Video as a teaching tool is a didactic tool that encourages more accessible and easily communicated education. Thus, it is concluded that, in the production of educational products in health, skills, competences and recognition of social needs are essential, as is the use of information and communication technologies in the most diverse ways. The Rational Use of Medicines is a relevant topic and lacks wide dissemination.
\end{abstract}

Keywords: Rational use of medicines; Educational products; Health education; Teaching. 


\section{Resumen}

Este artículo tiene como objetivo reportar la experiencia de construir productos educativos capaces de informar a los usuarios y profesionales de la salud sobre el Uso Racional de Medicamentos, a través de un estudio descriptivo, con enfoque cualitativo, del tipo informe de experiencia, resultado de la elaboración del Máster Profesional. en Educación en Salud y Tecnología en una Institución de Educación Superior en Alagoas. La construcción de productos educativos en salud es una de las estrategias didácticas utilizadas a lo largo del curso. Se creó una serie de tres videos sobre el Uso Racional de Medicamentos. El proceso de elaboración de estos productos educativos se basó en el método CTM3, a través del cual se exploraron las siguientes herramientas: Análisis Transaccional, Exploración Sensorial y Neurolingüística. El video como herramienta de enseñanza es una herramienta didáctica que fomenta una educación más accesible y de fácil comunicación. Así, se concluye que, en la producción de productos educativos en salud, son fundamentales las habilidades, competencias y el reconocimiento de las necesidades sociales, así como el uso de las tecnologías de la información y la comunicación en las más diversas formas. El Uso Racional de Medicamentos es un tema relevante y carece de amplia difusión.

Palabras clave: Uso racional de medicamentos; Productos educativos; Educación para la salud; Enseñando.

\section{Introdução}

A Organização Mundial de Saúde (World Health Organization, 1985), definiu que o uso racional de medicamentos ocorre quando pacientes recebem medicamentos apropriados às suas condições clínicas, em doses adequadas às suas necessidades individuais, por um período adequado e ao menor custo para si e para a comunidade.

Com a evolução dos medicamentos, além das vantagens no combate às doenças existem os problemas advindos de sua fabricação e utilização. As sobras de tratamentos anteriores, ou dispensação de medicamentos em quantidade superior ao tratamento devido à prescrição incompleta ou incorreta, juntamente com a impossibilidade de fracionamento de alguns desses produtos (Eickhoff, Heineck, e Seixas, 2009), podem causar problemas, tanto de saúde como ambientais, a partir do seu acúmulo nas residências, além da administração na própria residência, risco de intoxicação e do uso de medicamentos vencidos.

O descarte inadequado de medicamentos é outra questão a ser observada. De acordo com Falqueto e Kligerman (2013) aproximadamente $20 \%$ dos medicamentos utilizados pela população brasileira são descartados em lixo doméstico ou lançados na rede de esgoto.

O órgão responsável pela regulamentação do descarte desses medicamentos é a Agência Nacional de Vigilância Sanitária (ANVISA), que com base na Resolução da Diretoria Colegiada ${ }^{\circ} 222 / 18$ (Brasil, 2018), exige que estabelecimentos de serviços na área da saúde disponham de Plano de Gerenciamento de Resíduos de Serviço de Saúde (PGRSS). Contudo, não foram editadas normas que abranjam o consumidor final com relação ao descarte de medicamentos (Vaz, Freitas e Cirqueira, 2011).

Fatores como o aumento da expectativa de vida da população e o consequente aumento da carga de doença crônica, entre outros, influenciam a alta utilização de medicamentos. Estes são considerados importantes bens sociais. (Carvalho, Pascom, Souza Jr, Damacena e Szwarcwald, 2005).

Nesse sentido, a prática da automedicação é impulsionada por situações como, veiculação de propagandas de medicamentos isentos de prescrição na mídia, a presença da farmacinha caseira nos domicílios e a crença de que os medicamentos resolvem tudo (Arrais e Bertoldi, 2016). Em 1998, a Organização Mundial da Saúde (World Health Organization, 1998) define automedicação como a seleção e o uso de medicamentos sem prescrição ou supervisão do profissional prescritor.

No Brasil, o Sistema Nacional de Informações Tóxico Farmacológicas (Sinitox/Fiocruz) registrou cerca de 30 mil casos de intoxicações por uso de medicamentos. Embora não haver afirmações, é possível que esses casos tenham ocorrido em função de uso terapêutico errado, por prescrição médica incorreta e por automedicação. (Brasil, 2015).

Ramos et al. (2016) afirmam em seus estudos que a crescente complexidade da terapia medicamentosa, como o uso de quatro ou mais medicamentos, conhecida como polifarmácia, assim como de usuários com várias morbidades, elevam o risco 
de problemas relacionados à farmacoterapia, como eventos adversos e erros de medicação. Alguns estudos realizados com pacientes idosos demonstram que a prática clínica do farmacêutico corrobora para a melhoria da adesão ao tratamento, a redução do número de medicamentos utilizados e uma maior racionalidade das prescrições em longo prazo (Sáez Benito et al., 2013).

Dessa forma, é essencial ações que promovam educação em saúde e gerenciamento correto dos resíduos farmacêuticos de forma a minimizar o descarte inadequado dos medicamentos. Isso inclui medidas que incentivem o uso racional e fracionamento de medicamentos e ações como recolhimento e tratamento dos resíduos domiciliares, evitando-se que esses sejam descartados em lixos ou nas redes de esgoto. (Brasil, 2010; Falqueto e Kligerman, 2013).

Segundo a Organização Mundial da Saúde (World Health Organization, 2009), erro de medicação é qualquer evento evitável que pode causar ou induzir ao uso inapropriado de medicamento ou prejudicar o paciente, podendo ser considerado um Evento Adverso aos Medicamentos, quando gera danos ao paciente. Com o intuito de prevenir e reduzir a incidência de tais eventos adversos nos serviços de saúde, o Ministério da Saúde (MS) e a Agência Nacional de Vigilância Sanitária (ANVISA) publicaram, no ano de 2013, o Protocolo da Segurança na Prescrição, uso e Administração de Medicamentos, através da Resolução da Diretoria Colegiada n 36 (RDC 36), a ser praticada em todos os estabelecimentos que prestam cuidados à saúde, em todos os níveis de complexidade, em que medicamentos sejam utilizados para profilaxia, exames diagnósticos, tratamento e medidas paliativas. (BRASIL, 2013).

Este protocolo define que a administração segura de medicamentos depende de nove medidas chamadas de "9 certos". São eles: Paciente certo, Medicamento certo, Via certa, Hora certa, Dose certa, Registro correto, Orientação correta, Forma certa e Resposta Certa. Sendo cada um deles, uma etapa relacionada a administração segura da medicação.

O Sistema Único de Saúde (SUS) é orientador da formação de recursos humanos para a saúde. Embora o modelo tradicional, predomine o uso da lógica de transmissão vertical de conhecimento entre profissionais de saúde e público-alvo, faz-se necessário a prática dialógica, uma abordagem em que o processo de troca de saberes entre os participantes e a horizontalidade das relações sejam estimuladas e valorizadas (Figueiredo, Rodrigues Neto e Leite, 2010).

Segundo Coelho, Pinheiro e Magarinos Torres (2014), é essencial contar com a participação de diversos atores sociais na elaboração de ações para a promoção do uso racional de medicamentos (URM), como profissionais de saúde, gestores, educadores, controle social e usuários dos serviços.

O presente artigo tem por objetivo relatar a experiência do processo de construção de Recursos Educacionais, em vídeo, para aproximar seus usuários e profissionais de informações referentes ao armazenamento, descarte correto de medicamentos, alertar sobre os riscos da automedicação e demonstrar a importância da segurança na prescrição, uso e administração de medicamentos nos ambientes de saúde. Considerando que os medicamentos são insumos muito utilizados pela sociedade, geralmente são de alto custo e ainda representam sérios riscos pela utilização inadequada, percebe-se a importância de iniciativas que possam auxiliar na conscientização do seu uso racional.

\section{Metodologia}

Trata-se de um estudo descritivo, com abordagem qualitativa, do tipo relato de experiência (Pereira, Shitsuka, Parreira, Shitsuka, 2018) e nele estão descritas as etapas da construção de produtos educacionais elaborados a partir da dissertação do programa de Mestrado Profissional em Ensino na Saúde e Tecnologias de uma Instituição de Ensino Superior em Alagoas. O curso de mestrado da instituição tem em sua matriz curricular a disciplina Recursos Educacionais, onde os alunos são estimulados a produzirem produtos educacionais nas mais diversas subáreas da saúde, sendo um dos pré-requisitos para a conclusão do curso. 
Os referidos produtos educacionais são uma sequência de 3 vídeos que fazem parte de uma série voltados à Educação em Saúde na área do uso racional de medicamentos. Este artigo descreve o relato de experiencia na estruturação de produtos educacionais em forma de uma série de três vídeos. A intenção dos vídeos foi aproximar usuários da promoção do URM.

A elaboração de cada vídeo seguiu as seguintes etapas: concepção da ideia, estabelecimento do objetivo de cada vídeo, escolha do público-alvo e estabelecimento do orçamento. Em seguida foi definido o roteiro pela primeira autora, com elaboração da sequência de cenas e de falas. Depois foi elaborado um esboço sequencial de cenas, organizados em gráficos animados 2D que foram desenvolvidos pela autora 6, que também executou os demais passos: diagramação, narração, sonorização, créditos, edição e finalização dos vídeos.

A narração foi feita por voluntários, que documentaram a autorização para uso das vozes, sem que isso gerasse qualquer ônus para as autoras. As imagens e sons usados são livres de direitos autorais. Possuem licença Creative Commons (Atribuição - Não Comercial - Compartilhar Igual CC-BY-NC-AS).

As características técnicas dos vídeos estão descritas no Quadro 1. O formato do arquivo, Full HD, pretirwi ermite ampla divulgação e reprodução em diversos dispositivos.

Quadro 1 - Características técnicas dos vídeos.

\begin{tabular}{|l|c|c|c|}
\hline \multicolumn{1}{|c|}{ Título } & Duração & Tamanho & Arquivo \\
\hline Medicamentos, onde devem habitar? & 1 minuto e 50 segundos & 43 MB & MP4 \\
\hline Automedicação & 2 minutos e 53 segundos & 15 MB & MP4 \\
\hline Segurança na prescrição, uso e administração de medicamentos & 2 minutos e 41 segundos & 33 MB & MP4 \\
\hline
\end{tabular}

Fonte: Autores.

O desenvolvimento desses produtos educacionais baseou-se no que foi proposto por Bandeira (2009) para as produções em vídeo. O autor traz que essas produções precisam seguir um planejamento, com as seguintes etapas de produção: a) pré-produção: consiste no projeto do vídeo (ideia, objetivos, público-alvo, orçamento etc.), levantamento e pesquisa sobre o tema, roteiro, storyboard ou guia de registro; b) produção - registro das imagens, de acordo com a linguagem audiovisual; revisão do material audiovisual e, por fim, c) pós-produção - revisão do material audiovisual, roteiro de edição, edição do material audiovisual (uso de programa de edição), sonorização, créditos, títulos e grafismo, gravação de cópias e acabamento (capa e selo). O material elaborado também considerou o pensamento visual, transformando conceitos em imagens para auxiliar a compreensão (Almeida, 2007).

\section{Relato de Experiência}

O desenvolvimento dos vídeos foi iniciado através da pesquisa do termo uso racional de medicamentos (URM) e das suas implicações para a assistência farmacêutica. O URM está presente em políticas, guias, protocolos, documentos, artigos e livros relacionados à assistência farmacêutica, com ligação direta às atividades profissionais. Pensando no usuário de medicamentos e na equipe de trabalhadores da saúde em geral, foram escolhidos temas relevantes, capazes de orientar sobre o manejo, utilização e descarte seguro desses medicamentos. A partir dessa seleção de tema, a pesquisa foi concluída e os textos foram escritos. Após a escrita, os vídeos foram gerados utilizando os critérios propostos para sua construção.

De acordo com Santos e Warren (2020), é importante que todo produto educacional apresente um referencial teórico (embasamento sobre o tema abordado) e um referencial metodológico (definição do tipo de produto e sua respectiva 
estruturação). Deste modo, para a construção dos vídeos, foi adotado o Método CTM3 desenvolvido por Santos, Alves, Warren e Wyszomirska (2019), cuja finalidade é facilitar a inserção dos elementos que fariam parte da composição dos referidos produtos.

O método CTM3 é composto por três etapas distintas e baseado em três teorias, cuja aplicação nos produtos é definida pelos criadores do método como fundamental, em virtude da subjetividade e complexidade do ser humano, de suas ações e reações e da estrutura de sua personalidade. (Santos e Warren, 2020).

A primeira das fases é a Concepção do Produto (C), trata-se do planejamento, da definição do objetivo, do público a ser atingido, do meio utilizado para divulgação do produto. A segunda fase é o Referencial Teórico (T), consiste no levantamento de informações sobre a temática que se deseja abordar no produto em construção. Como terceira fase, Santos \& Warren (2020) propõem o Referencial Metodológico (M3).

O Referencial Metodológico propõe a abordagem de três teorias: a Análise Transacional, a Aplicação Multissensorial, e a Neurolinguística. A Análise Transacional trata da inserção de elementos facilitadores da comunicação envolvendo os três Estados de Ego da estrutura da personalidade: Pai, Adulto e Criança. De acordo com Cruz e Resende (2019), a Análise Transacional é um estudo psicodinâmico, enfatiza a modificação dos sentimentos, pensamentos e escolhas através do autoconhecimento e desenvolvimento pessoal tendo como objetivo uma linguagem simples, de fácil compreensão. A Aplicação Multissensorial concebe a inserção de elementos que evoquem a audição, visão, olfato, paladar/gustativo e tato/sinestésico, na perspectiva que os seres humanos possuem um potencial mais receptivo nas comunicações quando acessados por intermédio dos sentidos. A Neurolinguística trata da abordagem de aspectos subliminares da comunicação através da inserção das âncoras, que funcionam como elementos de reforço e resgate da memória original, e evocando o comportamento que se deseja despertar através do produto em questão. (Santos e Warren, 2020).

\section{Discussão}

Estudos mostram que as Tecnologias de Informação e Comunicação (TIC), como é o caso dos vídeos produzidos, provocam cada vez maior adesão e confiança na multiplicação de conteúdo, uma vez que permite a acessibilidade de grande parte da população (Pinto e Rocha, 2016). Esses produtos, quando criativos em sua construção, geram valorização do desenvolvimento de metodologias inovadoras, que auxiliam no desfecho de comunicação, saúde e comunidade. Com o desenvolvimento tecnológico cada vez mais rápido, e a evolução cada dia maior das mídias, a sociedade passou a ser rodeada por informação e comunicação que nos permitem um acesso ao mundo em tempo real como destacado por Nunes (2012) e Cavalcante, Silva, Rolim e Oliveira (2016). Em função disso optamos pelo uso de recursos audiovisuais leves que podem ser postados em mídias sociais e apresentam boa inserção em todas as camadas da população.

Segundo Caldas (2010), a democratização do conhecimento científico e a construção de espaços dialógicos entre a academia e a sociedade podem contribuir para o desenvolvimento de uma cultura científica, mas, sobretudo, possibilitar o exercício da cidadania. Para isso, é fundamental o desenvolvimento de estratégias que proporcionem a aproximação entre as diversas instituições que produzem o conhecimento científico e a realidade das comunidades, promovendo, assim, a democratização desses conhecimentos e a ampliação do diálogo entre os diferentes atores sociais.

Além disso, foi realizada a revisão das informações disponíveis na literatura científica sobre URM, objetivando garantir a base científica, a qualidade das informações e a confiança no conteúdo do material educativo. Tendo em vista a crescente onda de informações intencionalmente inverídicas foram realizadas pesquisas por meio das bases de dados das Ciências da Saúde como a Biblioteca Virtual em Saúde (BVS), o Portal Scientific Electronic Library Online (Scielo), a Literatura Latino-Americana e do Caribe em Ciências da Saúde (LILACS) e PubMed (Public/Publish Medline), com as palavras-chave selecionadas mediante consulta aos Descritores em Ciências da Saúde (DeCS) da Bireme: Uso Racional de 
Medicamentos. Recursos educacionais. Educação em Saúde para que todas as informações apresentadas nos vídeos pudessem ser referenciadas com base na literatura da área.

O principal entrave na construção dos vídeos foi a necessidade de adequação no tempo de cada um. Considerando que a quantidade de informações é vasta, e que os vídeos não devem ser tão extensos, pois possuem o objetivo de reter o interesse do telespectador no dispositivo. É importante aliar informação direta e objetiva a um curto espaço de tempo. Desta forma, optou-se pela produção de não apenas um vídeo, mas de três. Todos abordam o tema central URM, contudo a divisão do material em vários pequenos vídeos favorece sua divulgação e apreensão do conteúdo. Os vídeos foram intitulados: "Medicamentos, onde devem habitar?", “Automedicação" e "Segurança na prescrição, uso e administração de medicamentos". Assim, optou-se pelo formato MP4, pela facilidade de divulgação que este produto pode oferecer.

A escolha do tema e do recurso educativo se deu a partir de um olhar para a saúde da população e trabalhadores em saúde e sua relação com o uso de medicamentos, percebida pelos autores numa perspectiva de vivências técnicas diárias. Tal material foi desenvolvido buscando retratar questões do cotidiano de quem faz uso de medicamentos, desde sua utilização até o ato de descartar corretamente, colocando dúvidas ou situações com os quais eles poderiam se deparar. Objetivou-se que o recurso pudesse ser utilizado por profissionais da saúde em ações junto ao público-alvo, sendo esses profissionais mediadores do conhecimento numa perspectiva dialógica considerando principalmente fatores como nível de escolaridade. Na concepção, foi tomada a cautela de usar uma linguagem popular, que, representa grande relevância no processo de cuidado, pois o profissional é desafiado a buscar métodos de melhor interpretação ao seu público-alvo de forma individual, traduzindo o princípio do cuidar e educar. Baseado no estudo de Ferreira (2010) o conteúdo de materiais educativos deve colocar as informações para os indivíduos aliando teoria e prática, para que forneçam juntos um ensino-aprendizado científico e de vivência, facilitando a compreensão de determinado assunto.

De acordo com França, Rabello e Magnago (2019), esses recursos deixam de ser apenas um meio de Educação em Saúde e passam a ocupar com maior amplitude, o meio de comando da educação e do serviço em saúde, pois, através deles é possível verificar públicos vulneráveis e trabalhar sobre isso. De acordo ainda com Lima et al (2021), deve-se estimular o aperfeiçoamento nesta área de produção de recursos, para que esse acesso seja de qualidade, compreensível e palpável para todo o público, seguindo todos os princípios da ética e valorizando as diversas ferramentas tecnológicas que estão disponíveis atualmente.

Os vídeos foram construídos com roteiro criado pela primeira e segunda autoras, e possuem licença no Creative Commons, (Atribuição-Não Comercial-Compartilha Igual CC BY-NC-AS), com permissão para remixagem, adaptação e criação a partir do recurso para fins não comerciais, desde que atribuam aos autores o devido crédito e que licenciem as novas criações sob termos idênticos. Os temas que compuseram os vídeos e as suas principais características estão descritas abaixo (Quadro 2).

Quadro 2 - Detalhamento da composição dos vídeos.

\begin{tabular}{|l|l|}
\hline \multicolumn{1}{|c|}{ Vídeo } & \multicolumn{1}{c|}{ Descrição } \\
\hline $\begin{array}{l}\text { "Medicamentos, onde devem } \\
\text { habitar?" }\end{array}$ & $\begin{array}{l}\text { Reproduz um diálogo entre duas usuárias de uma Unidade de Saúde, debatendo sobre } \\
\text { orientações farmacêuticas sobre armazenamento e descarte de medicamentos. As duas } \\
\text { personagens, Rita e Nazinha, fluem uma conversa informativa sobre como o } \\
\text { indivíduo deve adotar hábitos que assegurem a integridade dos medicamentos que } \\
\text { precisam ser estocados em casa, bem como, quais atitudes devem tomar na hora de } \\
\text { descartar medicamentos vencidos sem prejudicar o meio ambiente. O estado de ego } \\
\text { Pai foi contemplado nos momentos de aconselhamento e orientação, onde uma das } \\
\text { personagens demonstra como o indivíduo deve proceder para proteger sua saúde e o } \\
\text { meio ambiente. Já o estado de ego Adulto, foi inserido nos momentos de reflexão da }\end{array}$ \\
\hline
\end{tabular}




\begin{tabular}{|c|c|}
\hline & $\begin{array}{l}\text { receptora da mensagem, onde ela reflete racionalmente sobre o assunto e esclarece } \\
\text { suas dúvidas de forma racional. Por último, o estado de ego Criança foi inserido } \\
\text { através de 'Paracetonildo', personagem que entra no contexto para ensinar de forma } \\
\text { lúdica os cuidados que devem ser tomados para garantir um bom armazenamento de } \\
\text { medicamentos. O personagem 'Paracetonildo' aparece também como âncora do } \\
\text { vídeo. }\end{array}$ \\
\hline "Automedicação" & $\begin{array}{l}\text { O vídeo em formato de aula, traz as informações importantes sobre os riscos da } \\
\text { automedicação e alerta sobre os principais perigos desta prática. Os três estados de } \\
\text { ego foram inseridos de forma semelhante ao primeiro vídeo produzido, com a } \\
\text { diferença de que não há um diálogo, e sim uma orientação junto a momentos de } \\
\text { reflexão, onde são contemplados os estados de ego Pai e Adulto. O estado de ego } \\
\text { criança aparece através do formato de desenho animado das imagens do vídeo. A } \\
\text { exploração sensorial neste vídeo, fica mais evidente no sentido cinestésico, uma vez } \\
\text { que foram inseridas figuras que representam situações desagradáveis que podem ser } \\
\text { vivenciadas nos casos de automedicação. Dentre os outros sentidos, audição e visão } \\
\text { também são estimuladas pela forma de vídeo, e olfativo e gustativo, aparecem através } \\
\text { das ações de administração e tomada de medicamentos, aparecendo através de ações } \\
\text { que os representam. Neste material, temos o farmacêutico como âncora, lembrando da } \\
\text { importância deste profissional no contexto e gerando alertas aos expectadores, } \\
\text { principalmente promovendo a educação e o Uso Racional de Medicamentos. }\end{array}$ \\
\hline $\begin{array}{l}\text { "Segurança na prescrição, uso e } \\
\text { administração de medicamentos" }\end{array}$ & $\begin{array}{l}\text { Com formato de imagens semelhante à do segundo vídeo, mantendo fundo de tela } \\
\text { preto, destaque nas ações e formato de aula - alerta. Traz uma aula sobre segurança } \\
\text { na prescrição, uso e administração de medicamentos, explicando cada etapa que } \\
\text { confere ao ato. Aborda os chamados "9 certos", correspondentes a } 9 \text { atitudes que } \\
\text { devem ser praticadas para garantir a segurança do paciente em relação aos } \\
\text { medicamentos. Os três estados de ego foram inseridos no vídeo, com ênfase ao Pai e } \\
\text { Adulto, sendo o Adulto que explica o que é o tema explorando a razão, e o Pai, o que } \\
\text { aconselha, determinando o que deve ser feito, uma vez que o tema exige um maior } \\
\text { teor de informações e atitudes relacionadas à responsabilidade. O estado de ego } \\
\text { Criança é representado pelas imagens de desenho animado. Os sentidos mais } \\
\text { evidentes neste material são audição e visão, e por ser uma continuidade da série, o } \\
\text { vídeo também explora o cinestésico e os outros sentidos, olfativo e gustativo, } \\
\text { aparecem através das diversas formas farmacêuticas (oral, nasal) representadas pelas } \\
\text { imagens do vídeo. Novamente temos um 'boneco cápsula' como âncora, com } \\
\text { autofalante, chamando atenção para o assunto abordado. }\end{array}$ \\
\hline
\end{tabular}

Fonte: Autores.

No referencial metodológico, foi seguida a teoria do método CTM3, baseado em: Análise Transacional utilizando a ferramenta da estrutura de personalidade com os três Estados de Ego, Exploração Sensorial usando os cinco sentidos e Neurolinguística usando a ferramenta âncoras.

Na concepção de produtos educacionais em saúde, ruídos de comunicação devem ser evitados. Deve-se estruturar o conteúdo de maneira que ele seja de fácil entendimento. De acordo com Silva, Franco, Rodrigues, Coelho e Bezerra (2015), o nível educacional reduzido dos usuários do SUS pode ser um reflexo da sua baixa condição socioeconômica. Assim, o autor reforça a importância de recursos adequados às particularidades de cada população. Os diálogos entre personagens em produtos educacionais em saúde vêm sendo utilizado como estratégia facilitadora da compreensão do conteúdo abordado (Cordeiro et al., 2017). 
Os vídeos foram validados por banca de defesa e disponibilizados em mídias sociais para ampla divulgação e informação. Estão hospedados no portal EduCAPES e, também, publicados no canal Youtube da Instituição de Ensino. Foram aplicados em Unidades Básicas de Saúde e compartilhados com equipe de saúde e usuários do SUS.

O endereço do primeiro vídeo intitulado "Medicamentos, onde devem habitar?" é http://educapes.capes.gov.br/handle/capes/429442, o segundo com o tema sobre “Automedicação" tem o link http://educapes.capes.gov.br/handle/capes/584841, e o último vídeo que versa sobre "Segurança na prescrição, uso e administração de medicamentos”, possui o link http://educapes.capes.gov.br/handle/capes/584830.

O papel do telespectador, na visão do público-alvo, é de suma importância na validação de um produto educacional fornecendo informações para que os autores verifiquem se o objetivo do material foi alcançado e quando não, que ele possa ser readequado conforme reais necessidades de seu público (Neto, Caetano, Barros, Silva e Vasconcelos, 2017).

\section{Considerações Finais}

O uso racional de medicamentos está ligado a adequadas condições de armazenamento e descarte. Grande parte da população realiza essas práticas de maneira inadequada, por falta de informação sobre danos causados ao meio ambiente e à saúde pública. A prática da automedicação deve ser evitada, prevendo a segurança do paciente. A população e os profissionais de saúde necessitam de recursos versáteis nesta busca de informações.

Na produção de produtos educacionais em saúde, as habilidades e o reconhecimento das necessidades sociais são primordiais, assim como o uso das tecnologias da informação e comunicação. Os vídeos têm uma importância singular na contribuição para a construção de vínculos entre pacientes e profissionais de saúde, facilitando o processo educacional através de uma comunicação dialógica, com objetivo de troca de saberes para facilitar mudanças de comportamentos, autonomia e melhoria na qualidade de vida.

Espera-se, portanto, a geração de impacto no público, que receberá as informações sobre o assunto, a continuidade da transmissão de conhecimento e a criação de novos trabalhos em prol da promoção da saúde coletiva e do bem-estar ambiental. E por último, não menos importante, sugere-se novas pesquisas na área, com a utilização de vídeos como instrumento facilitador em processos educacionais, sendo produzidos em diversos cenários, com variabilidade de temas, bem como, em diferentes níveis de atenção à saúde.

\section{Referências}

Almeida, D.M. (2017). Elaboração de materiais educativos. Escola de Enfermagem da Universidade de São Paulo. https://edisciplinas.usp.br/pluginfile.php/4412041/mod_resource/content/1/ELABORA\%C3\%87\%C3\%83O\%20MATERIAL\%20EDUCATIVO.pdf

Arrais, P. S. D. e Bertoldi, A. D. (2016). Prevalência da automedicação no Brasil e fatores associados. Revista Saúde Pública, 50(2), 1-11.

Bandeira, D. (2009). Materiais Didáticos. Curitiba: IESDE.

Brasil. Ministério da Saúde. (2013). Protocolo de Segurança na prescrição, uso e administração de medicamentos. Brasília.

Brasil (2015). Fundação Oswaldo Cruz/Centro de Informação Científica e Tecnológica/Sistema Nacional de Informações Tóxico-Farmacológicas. Rio de Janeiro.

Caldas, G. (2010). Divulgação e relações de poder. Informação \& informação, 5(1), 31-42.

Carvalho, M. F., Pascom, A. R. P., Souza Júnior, P. R. B., Damacena, G. N., e Szwarcwald, C. L. (2005). Utilization of medicines by the Brazilian population, 2003. Cadernos de Saúde Pública, 21(1), 100-108.

Cavalcante, L. E., Silva, A. R. S., Rolim, R. M. e Oliveira, L. R. (2016). Recursos Educacionais Abertos na Educação Superior e Promoção da Saúde. In J. Sánchez (Org), Nuevas Ideas en Informática Educativa, (pp. 286 - 293). Santiago de Chile.

Coelho, H. L. L., Pinheiro, R. M., \& Magarinos-Torres, R. (2014). Promoção do uso racional de medicamentos. In C. G. S. Osório de Castro, Luiza, V. L., Castilho, S. R., Oliveira, M. A., e Jaramilho, N. M. (org). Assistência Farmacêutica - gestão e prática para profissionais da saúde (pp. 283-294). Rio de Janeiro: Editora Fiocruz. 
Cordeiro, L. I., Lopes, T. O., Lira, L. E. A., Feitoza, S. M. S., Bessa, M. E. P., Pereira, M. L. D., Feitoza, A. R., e Souza, A. R. (2017). Validação de cartilha educativa para prevenção de HIV/Aids em idosos. Revista Brasileira de Enfermagem, 70(4), 775-82.

Cruz, M. U. S., e Resende, S. M. (2019). Análise Transacional e os estados de ego: uma revisão bibliográfica. Revista Eletrônica da Reunião Anual de Ciências, 9(1).

Eickhoff, P., Heineck, I., e Seixas, L. J. (2009). Gerenciamento e destinação final de medicamentos: uma discussão sobre o problema. Revista Brasileira de Farmácia, 90(2), 64-68.

Falqueto, E., e Kligerman, D. C. (2013). Diretrizes para um Programa de Recolhimento de Medicamentos Vencidos no Brasil. Ciência Saúde Coletiva, 18(3).

Ferreira, E. C. (2010). O Uso dos Audiovisuais como Recurso Didático. (Dissertação de Mestrado). Universidade do Porto, Porto.

Figueiredo, M. F. S., Rodrigues Neto, J. F., e Leite, M. T. S. (2010). Modelos aplicados às atividades de educação em Saúde. Revista Brasileira de Enfermagem, 63(1), 117-121.

França, T., Rabello, E. T. e Magnago, C. (2019). As mídias e as plataformas digitais no campo da Educação Permanente em Saúde: debates e propostas. Saúde debate, 43(spe 1), 106-115.

Lei $n^{o}$. 12.305, de 02 de agosto de 2010. Institui a Política Nacional de Resíduos Sólidos, e dá outras providências. http://www.planalto.gov.br/ccivil_03/_ato2007-2010/2010/lei/112305.htm

Lima, M. A. G., Mendes, L. S. F., Machado, A. L. L. B., Freitas, M. C., Santos, T. R., Bezerra, A. D. C., Gomes, F. T. B., Feitosa, K. C. S., Nascimento, C. E. M., Marçal, M. E. A., Silva, V. C., e Silva, L. S. (2021). Impacto das mídias sociais nas ações de educação em saúde voltadas à população. Research, Society and Development, 10(2), 1-7.

Neto, N. M. G., Caetano, J. A., Barros, L. M., Silva, T. M. e Vasconcelos, E. M. R. (2017). Primeiros socorros na escola: construção e validação de cartilha educativa para professores. Acta Paulista de Enfermagem, 30(1), 87-93.

Nunes, S. M. S. (2012). O vídeo na sala de aula: um olhar sobre essa ação pedagógica. (Monografia de Especialização), Universidade Federal do Amapá, Macapá.

Pereira, A. S., Shitsuka, D. M., Parreira, F. J., e Shitsuka, R. (2018). Metodologia da Pesquisa Científica. UFSM.

Pinto, L. F., e Rocha, C. M. F. (2016). Inovações na Atenção Primária em Saúde: o uso de ferramentas de tecnologia de comunicação e informação para apoio à gestão local. Ciênc. saúde coletiva, 1(5), 1433-1448.

Ramos L. R., Tavares, N. U. L., Bertoldi, A. D., Farias, M. R., Oliveira, M. A., Luiza, V. L., Pizzol, T. S. D., Arrais, P. S. D., e Mengue, S. S. (2016). Polifarmácia e polimorbidade em idosos no Brasil: um desafio em saúde pública. Revista Saúde Pública, 50(supl 2), 1s-9s.

Resolução da Diretoria Colegiada n 222, de 28 de março de 2018. Regulamenta as Boas Práticas de Gerenciamento dos Resíduos de Serviços de Saúde e dá outras $\quad$ providências. https://www.cff.org.br/userfiles/file/RDC\%20ANVISA\%20N\%C2\%BA\%20222\%20DE\%2028032018\%20REQUISITOS\%20DE\%20BOAS\%20PR\%C3\%81 TICAS\%20DE\%20GERENCIAMENTO\%20DOS\%20RES\%C3\%8DDUOS\%20DE\%20SERVI\%C3\%87OS\%20DE\%20SA\%C3\%9ADE.pdf

Sàez Benito, L., Fernandez Llimos, F., Feletto, E., Gastelurrutia, M. A., Martinez Martinez, F., e Benrimoj, S. I. (2013). Evidence of the clinical effectiveness of cognitive pharmaceutical services for aged patients. Age Ageing, 242(4), 442-449.

Santos, A. A., Alves, C. F., Warren, E. M. C., e Wyszomirska, R. M. A. F. (2019). Integrated Modal of Course Based on Edu-Communication and PsychoCommunication in Learning. Creative Education, 10(6), 1080-1090. https://file.scirp.org/pdf/CE_2019061115430264.pdf.

Santos, A. A., e Warren, E. M. C. (2020). Método CTM3 como dispositivo de ensino, aprendizagem e comunicação em produtos educacionais. In: A. A. Santos (org). Educação em saúde: trabalhando com produtos educacionais. (pp. 13-28). Maceió: Editora Hawking.

Silva, A. N. B., Franco, E. S., Rodrigues, E. M., Coelho, M. O., e Bezerra, J. C. P. (2015). Elaboração de material didático para educação em saúde direcionado para hipertensão arterial. Saúde (Santa Maria), 41(1), 175-184.

Vaz, K. V., Freitas, M, M., e Cirqueira, J. Z. (2011). Investigação sobre a forma de descarte de medicamentos vencidos. Cenarium Pharmaceutico, 4(4), 6-27.

World Health Organization (1985). The Rational use of drugs: report of the conference of experts.Nairóbi. Geneva: WHO.

World Health Organization. (1998). The role of the pharmacist in self-care and self-medication. Recuperado em 20 de fevereiro, 2021 de http:// apps.who.int/medicinedocs/pdf/whozip32e/ whozip32e.pdf.

World Health Organization. (2009). World Alliance for Patient Safety, Taxonomy: The Conceptual Framework for the International Classification for Patient Safety: final technical report. https://www.who.int/patientsafety/taxonomy/icps_full_report.pdf. 